\title{
Automatic Detection of Features (Markers) on a Three-Dimensional Model of a Human Face
}

\author{
Witold Stankiewicz and Michał Rychlik \\ Poznan University of Technology, Division of Virtual Engineering, Poland \\ witold.stankiewicz@put.poznan.pl \\ http://virtual.edu.pl
}

\begin{abstract}
The post-processing and correlation analysis (like Proper Orthogonal Decomposition) requires the same topology for all objects in the database. Thus, in the case of 3D scanned data, registration is required. One of possible choices is elastic registration based on the known positions of certain markers (features) on the surface of each scanned object.

The present paper targets the method of automatic detection of such markers on the scanned human faces and the elastic deformation resulting in the same topology of the triangular meshes after the registration. Resulting data might be analyzed using methods like POD.
\end{abstract}

Keywords: data registration, 3D scanning, POD, PCA.

\section{Introduction}

The increasing globalization, growth of tourism and labor migration affect the increase in passenger traffic as well as increasing frequency of terrorist threat alerts. The challenges of the modern world cause increased demand for the systems of auto-matic recognition and identification of individuals.

Such a recognition might be based on the individual anatomical / physiological features or on the basis of persons behavior. Commonly used biometric methods are based among others on the retinal, fingerprint, hand, dental or facial identification, as well as voice or signature analysis.

Face recognition, based so far on flat photographs and analysis of eigenfaces, in-creasingly relies on the spatial data coming from three-dimensional scanning.

Point cloud and resulting from the following processing computational mesh may consist of the large number of degrees of freedom. The database containing several thousand objects might require processing the data of the order of $10^{9}$, which obviously causes great difficulties in the storage and processing. Therefore, it is important to use methods reducing the size (dimensionality) of the data while maintaining high accuracy of the mapping of full spatial information about the object. The methods used to reduce and simplify the database and thus making an analysis of geometrical features possible are modal analysis.

The paper targets the method of automatic detection of the most important markers on the human face, basing on the curvature of the facial surface. Such markers, used together with the definition of the facial surface's boundary and 
mesh deformation algorithms, constitute elastic registration technique, resulting in the mesh defining the geometry of new object and remaining the topology (number of nodes, element connectivity) of base mesh.

The paper is organized as follows. In the next, second section the data acquisition based on 3D scanning is described. Next, The Proper Orthogonal Decomposition and it's requirements on the input data are briefly introduced. The algorithm of automatic detection of the markers is described in fourth section and the elastic registration method is summarized in section 5. Finally, the whole approach is summarized.

\section{3D Scanning}

The input data for numerical experiment and biometric PCA/POD analysis has been obtained using 3D optical, structural light scanner (fig. 1). In this work the geometry for more than 100 human faces of different persons with neutral expression has been acquired.
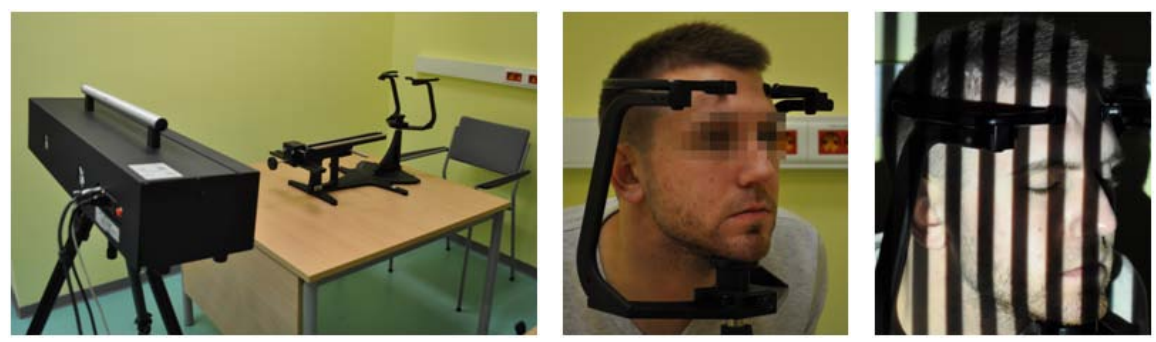

Fig. 1. Left: 3D structural light scanner. Middle: face setting with positioner. Right: scanning using the measurement stripes

The following procedure of 3D scanning and post-processing has been used: each input object has been scanned from three directions: front and both (left and right) profiles (fig. 2, left-middle). Left and right profiles were measured from the angles $-30^{\circ}$ and $45^{\circ}$ to the sagittal plane, respectively.

After the scanning process the three separate point clouds have been merged into one, single point cloud. In the next steps the $3 \mathrm{D}$ surface model has been computed basing on the point cloud and an individual polygonal surface mesh (containing 50k triangles, instead of few markers from "golden triangle" area) has been created (fig. 2, right).

\section{Data Registration for Modal Decomposition}

One of the most popular methods of modal analysis is Proper Orthogonal Decomposition (POD), also known as Principal Component Analysis (PCA) [12, 

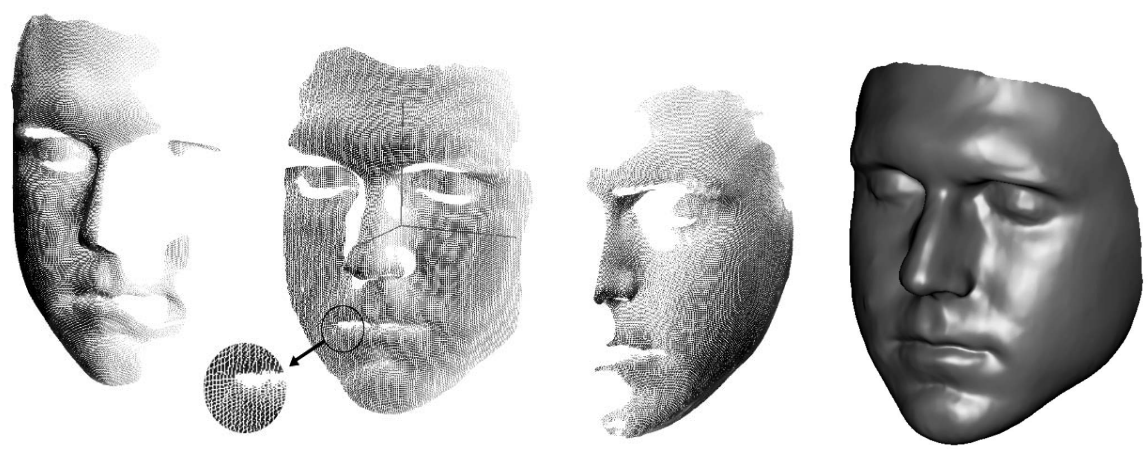

Fig. 2. Scanning of human face: set of three basic point clouds of the face (right, front and left profile, total ca. 500k mln points) and resulting surface model of the face (ca. $50 \mathrm{k}$ triangles). White spots represent the areas not illuminated while scanning at a given angle

as well as its variations like Snapshot method, Kernel PCA [3], Multilinear PCA 4 and Generalized POD. In this family of methods, the modal information is obtained from the solution of an eigenproblem for auto-correlation matrix based on input snapshots.

PCA is based on the assumption, that the snapshots $v_{i}$ containing data (here: geometry) for successive obects are correlated.

The first step is the computation of time-averaged solution $\bar{u}$ and the centering of the snapshots by the subtraction $\hat{v}_{i}=v_{i}-\bar{u}$.

Resulting vectors $\hat{v}_{i}$ describe the differences between the snapshots and the mean geometry. This data is required to compute the autocorrelation matrix $C$ :

$$
C=\frac{1}{M} S S^{T}, \quad \text { where } \quad S=\left[\dot{v}_{1}, \dot{v}_{2}, \ldots \dot{v}_{M}\right] .
$$

Eigenvectors $u$ of standard eigenproblem $C u=\lambda I u$, related to eigenvalues $\lambda$ of largest magnitude, are the most dominant PCA/POD modes.

A necessary prerequisite to carry out the modal decomposition is to preserve the same topology for all input data. In particular, the same number of nodes and mesh connectivity is required. The processing of raw input data to obtain the same topology for all input objects is called data registration. Unfortunately, the human face is characterized by a quite complex geometry, that makes 3D registration difficult.

\section{Curvature-Based Feature Detection}

In order to solve the problem of registration of data from $3 \mathrm{D}$ scanning, the program automatically detecting the characteristic points (markers) on the surface of the face has been developed. The algorithm of the program is as follows: triangular mesh (stored in Wavefront OBJ format) is cut by the designated plane 
(parallel to the axis of the coordinate system, such as $\mathrm{XZ}$ or YZ), which leads to the formation of a new curve. After an initial sorting of the vertices lying on the curve, it is possible to rotate it around the axis normal to the aforementioned surface. After the (optional) rotation, the extremes of the (parametric) function defining the curve are calculated.

Particular features (markers) on the face are searched using various cutting planes, rotations and extremes, in the following order.

\subsection{Apex (Tip) of the Nose}

The face is cut by a $Y Z$-plane at coordinate $X=0$ (symmetry plane, fig 3 , left). The intersection of the facial surface and the cutting-plane is a curve, on which maximal $Z$ value is searched. This point is used as the location where second cutting-plane, parallell to $X Z$-plane, is placed (fig, 3 right).

Again, maximum value of $Z$ coordinate is searched on the intersecting curve. This approach, possible to be repeated iteratively, allows the correction of the first marker's position and is especially important in the case of distortion of the nose.
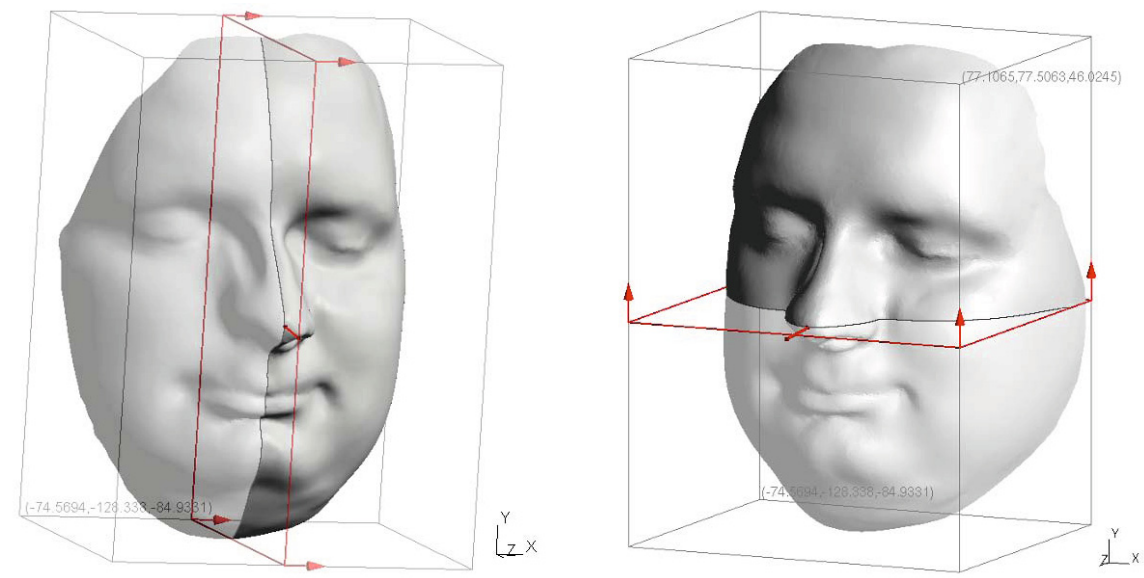

Fig. 3. Marker detection - apex of the nose. For the visualisation of meshes, markers and cutting planes, gmsh tool $[8]$ is used

\subsection{Nasal Bridge (Root, Nasion) and the "End" of the Nose}

Second marker is defined as a mid-point of the nasal bridge - a point on the forehead between the eyes (fig 4, left). It is detected using the first intersection curve $(Y Z$-plane at coordinate $X=0)$. Approximate position of this point is defined as the point lying above the $Y$-coordinate of nose tip, where the $Z$ coordinate is minimal. Again, iterative correction of the coordinates using various cutting-planes is possible. 
Third marker defines the point between the philtrum and columella, where the nasolabial angle is measured. Again, it's approximate position is detected on the same curve as the second marker. This time, the first local minimum of the $Z$-coordinate below the apex of the nose is searched (fig 4 left).
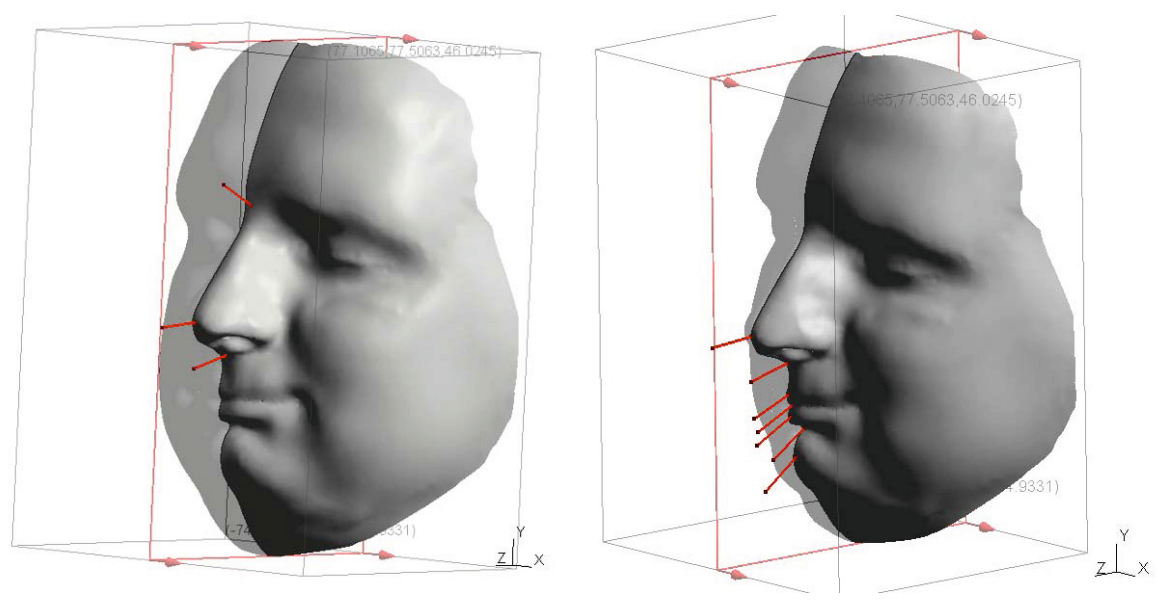

Fig. 4. Marker detection - bridge and "end" of the nose

\subsection{Lips and Chin}

The curve lying on the intersection of the surface and $Y Z$-plane is used in the detection of a few another points (fig, right).

Fourth marker, defining the "center of the mouth", is located in the second minimum of the $Z$-coordinate, considering the points below the apex of the nose.

Fifth and sixth markers, lying on the top and bottom lines of the lips, are defined as two local maxima of $Z$-coordinate lying in the vicinity of the fourth marker.

Another two points lying on the aforementioned curve approximate the coordinates of markers 7 and 8, lying on the chin. These are, respectively, the points of the biggest depression and maximal protuberance.

\subsection{Correction of the Third Marker's Position}

After determining the coordinates of the markers 4-6 defining the lips, the position of the marker defining "end" of the nose is corrected. Previously used curve is rotated by 45 degrees (fig. [5), then the lowest point between the first and fifth marker is searched. 


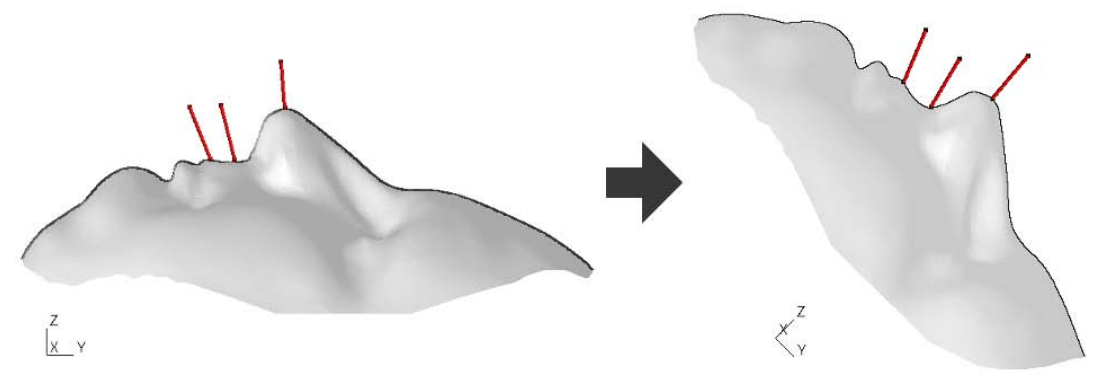

Fig. 5. Correction of the position of the "end" of the nose

\subsection{Width of the Mouth}

For the rest of the markers, horizontal cutting-planes are used for initial position estimation. In the case of markers 9 and 10, defining the width of the mouth, the $Y$-coordinate of $X Z$-plane is set on the value of the fourth marker, defining the "center of the mouth" (fig[6). The resulting intersection curve is analysed in both directions, starting from the marker 4 ("center of the mouth"). New markers' initial positions are the local minima of $Z$-coordinate. These positions are adjusted in the iterative search of smallest $Z$-coordinate in the vicinity of the initial location.

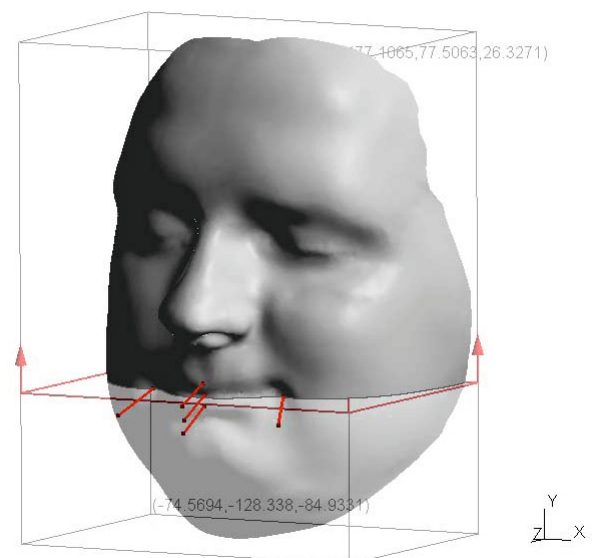

Fig. 6. Search for the markers defining the width of the mouth

\subsection{Width of the Nose}

Markers 11 and 12, defining the width of the nose, are initially found on the curve resulting from the intersection of facial surface and $X Z$-plane on marker 1 (apex of the nose), as shown on the left of the fig 7 These approximate positions are 
corrected by the search - in the close vicinity of initial location - of the maximum or minimum of $X$-coordinate, respectively (fig, 7 right). If this approach fails, the curve is rotated about $Y$-axis and iterative procedure is repeated.

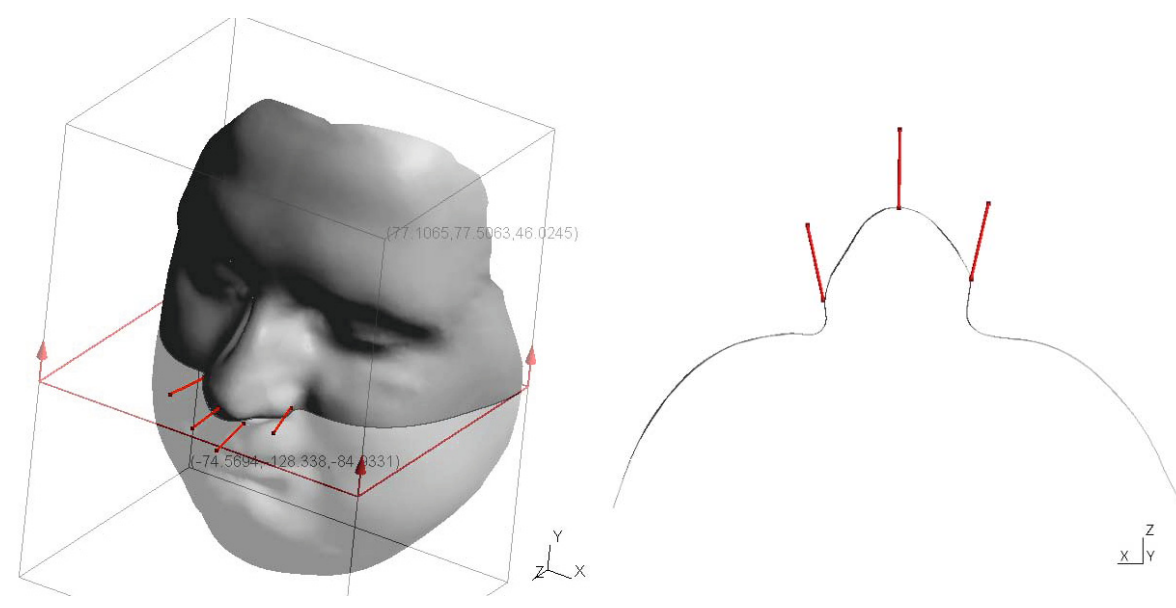

Fig. 7. Detection of the markers defining width of the nose

\subsection{Eyes}

The last four markers are placed in the corners of the eyes, as depicted in fig 8 They are approximately defined by the local minima of $Z$-coordinate on the intersection of facial surface and $X Z$-plane at $Y=0$ (position of eyes after rigid registration). Next, these initial positions are adjusted using the techniques mentioned before: use of additional cutting-planes, rotations and searches in the neighbouhood on the given point.

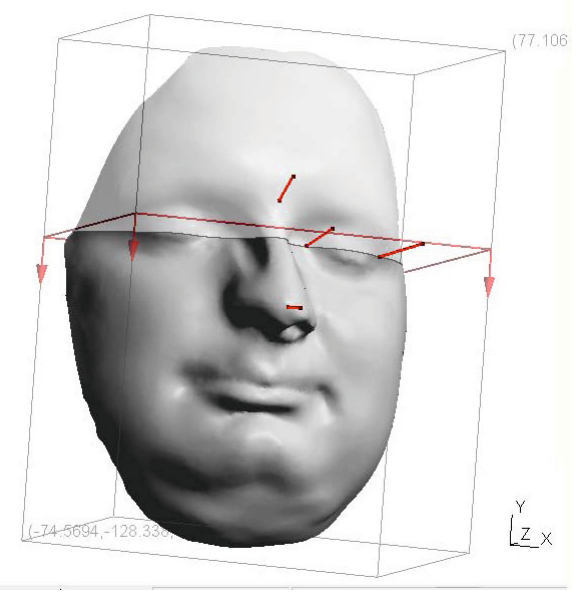

Fig. 8. Detection of the markers defining width of the eyes 


\section{$5 \quad$ Elastic Registration of Human Faces}

As soon as the definition of the boundary curves (face contour) and the coordinates of the markers are known, elastic registration of the 3D data might be performed. Principal Component Analysis requires the same topology for all the objects in the input database. This prerequisite might be achieved by the use of one, base mesh for all the individuals in the database, deformed such a way, that the nodes of that mesh lay on the surface of a given individual face.

This might be done by mesh deformation method, e.g. basing on spring analogy (using Finite Element Method [7]) or Inverse Distance Weighting [6]5] .

In this method, the markers of the reference face (base mesh) are snapped to the corresponding markers on the registered mesh and the coordinates of the remaining nodes from surrounding areas are interpolated basing on the weighting functions $w_{i}$ :

$$
x^{\kappa}=\sum_{i=1}^{n} x_{i} w_{i}
$$

where

$$
w_{i}=\frac{d_{i}^{-p}}{\sum_{j=1}^{n} d_{j}^{-p}} .
$$

In the above equations $p$ is an arbitrary positive real number, $n$ is a number of markers and nodes on boundary curves and $d_{i}\left(d_{j}\right)$ is a distance between interpolated and $i$-th $(j$-th) known coordinate.

In the finishing stage, for all the nodes translated using Inverse Distanse Weighting the nearest neighbours on the surface of new (registered) face are searched using kd-tree algorithm [9, and the coordinates of these nodes are adjusted so as to lie on the surface of a new face.

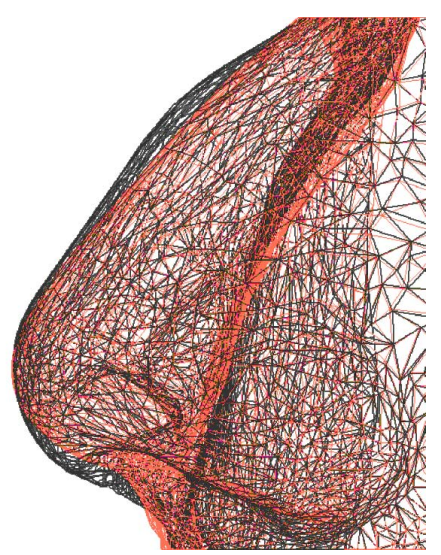

Fig. 9. The base mesh of the nose in the position of registered face, before finishing stage and after it (right) 

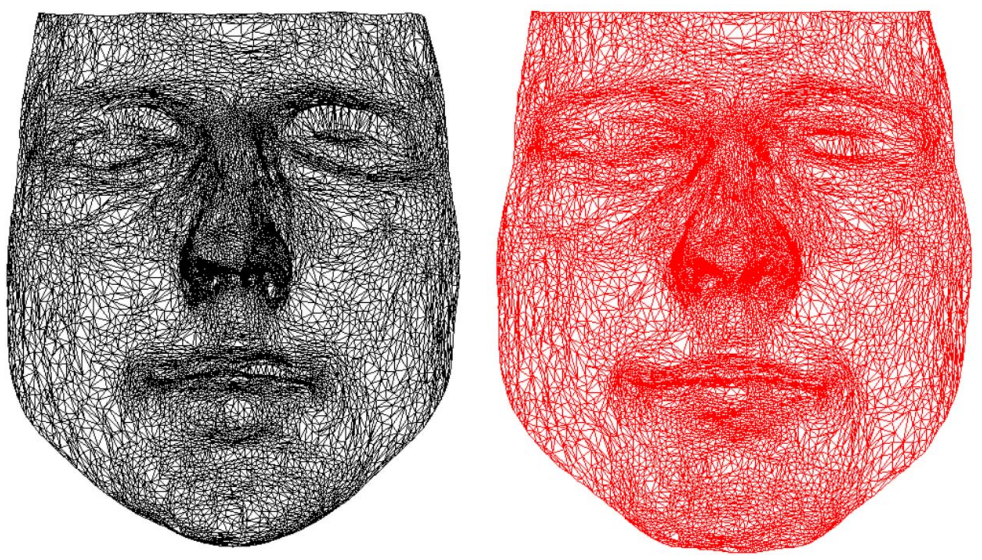

Fig. 10. Base mesh of the reference face (left) and the deformed base mesh stretched to the mesh of registered face (right)

This step leads to a better matching of the meshes in the areas that are not described by the characteristic points, such as the cheeks and forehead, and the areas lying between the markers - for example, the curvature of the nose (fig 9).

The example of whole 3D registration procedure is depicted in the fig 10 . where the same, base mesh is representing reference face (left) and registered face (right).

\section{Summary}

The effect of accurate registration is a deformed mesh of the reference face, describing the geometry of the registered face, and introduced into the database.

In the present paper a method for automatic detection of characteristic points (markers) on the surface of the face is described. This method allows to perform accurate registration (eg elastic) of scanned face, leading to a triangular mesh of the same topology as in the case of previous (base) mesh.

This approach is a key enabler of further analysis of scanned faces, for example using statistical analysis or moda decomposition using Principal Component Analysis.

\section{References}

1. Lumley, J.L.: The structure of inhomogeneous turbulent flows. Atmospheric Turbulence and Radio Wave Propagation, 166-178 (1967)

2. Rychlik, M., Stankiewicz, W., Morzynski, M.: Application of modal analysis for extraction of geometrical features of biological objects set. In: BIODEVICES 2008: Proc. 1st Int. Conf. Biomed. Electronics and Devices, vol. 2, pp. 227-232 (2008)

3. Hoffmann, H.: Kernel PCA for Novelty Detection. Pattern Recognition 40, 863-874 (2007) 
4. Lu, H., Plataniotis, K.N., Venetsanopoulos, A.N.: MPCA: Multilinear principal component analysis of tensor objects. IEEE Trans. Neural Netw. 19(1), 18-39 (2008)

5. Stankiewicz, W., Roszak, R., Morzynski, M., Noack, B.R., Tadmor, G.: Continuous Mode Interpolation between Multiple Operating and Boundary Conditions for Reduced Order Modelling of the Flow. AIP Conference Proceedings 1389(1), 94-97 (2011)

6. Shepard, D.: A two-dimensional interpolation function for irregularly-spaced data. In: Proceedings of the 1968 ACM National Conference, pp. 517-524 (1968)

7. Dhondt, G.: The Finite Element Method for Three-Dimensional Thermomechanical Applications. Wiley (2004)

8. Geuzaine, C., Remacle, J.F.: Gmsh: A 3D finite element mesh generator with built in pre and post processing facilities. International Journal for Numerical Methods in Engineering 79(11), 1309-1331 (2009)

9. Kennel, M.B.: KDTREE 2: Fortran 95 and C++ software to efficiently search for near neighbors in a multi-dimensional Euclidean space. arXiv preprint physics/0408067 (2004) 